\title{
Pot Pourri - A Bundle of Findings
}

\section{JS Rajkumar ${ }^{* 1}$, Akbar S ${ }^{2}$, Anirudh Rajkumar ${ }^{2}$, Hema Tadimari ${ }^{2}$ and Vijay Vendra $P^{2}$}

${ }^{1}$ Chief surgeon, Lifeline institute of minimal access surgery

${ }^{2}$ Assistant surgeon, Lifeline institute of minimal access surgery

*Corresponding author: Akbar S, Chief surgeon, Lifeline institute of minimal access surgery, India, Tel: +919566124322, E-mail: sm_akbars@yahoo.co.in

Citation: JS Rajkumar, Akbar S, Anirudh Rajkumar, Hema Tadimari, Vijay Vendra P (2017) Pot Pourri - A Bundle of Findings. J Aids Hiv Inf 3(2): 203. doi: 10.15744/2454-499X.3.203

Received Date: October 12, 2016 Accepted Date: December 28, 2017 Published Date: December 29, 2017

List of Abbreviations: TEP: Total extra peritoneal hernioplasty; SEMS: self-expanding metallic stent; PTBD: percutnaeous transhepatic biliary drainage; CBD: common bile duct

Keywords: HIV; Multiple Surgeries; X Ray

\section{Discussion}

This 63 year old gentleman, known to suffer from retroviral disease for the past 12 years, first presented in 2011 with carcinoma of caecum, for which he underwent a radical right hemicolectomy. Following this, he presented with descending colon malignancy for which completion colectomy with ileo sigmoid anastomosis was performed (2013). In 2014 he had a right inguinal hernia for which total extraperitoneal hernioplasty was done (TEP) and a mesh tacked with the structures in the posterior wall of inguinal canal. Subsequently in 2016 he developed gastric outlet obstruction secondary to nodal metastasis from his previous malignancy, which was treated with gastrojejunal bypass. He also had a percutaneous transhepatic biliary drainage (PTBD) inserted at that time for obstructive jaundice. After 6 weeks, through the PTBD, an extra long metallic self expanding stent (SEMS) was internalized in the common bile duct (CBD) all the way upto the duodenum (upto D3). Later the PTBD was removed. At this point of time, despite a double colic malignancy with nodal metastasis, with the background of HIV, the patient continues to be stable, anicteric, with no vomiting, but progressively growing weaker due to his malignancy. This $\mathrm{x}$-ray is presented because of the details that can be seen in it:

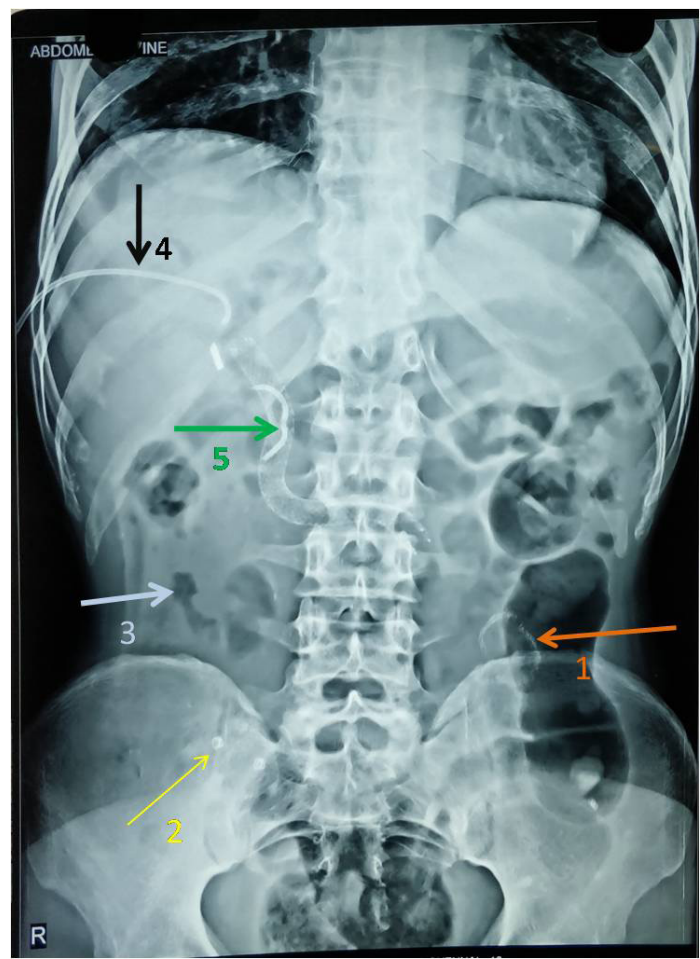


1. Circular stapler shadow indicating stapled ileo sigmoid anastomosis

2. Tackers in the right inguinal region indicating mesh placement

3. Absence of bowel gas in the right lower quadrant indicating right colectomy

4. PTBD in the right upper quadrant

5. SEMS that can be seen going across D3

\section{References}

1. Long JL, Engels EA, Moore RD, Gebo KA (2008) Incidence and outcomes of malignancy in the HAART era in an urban cohort of HIV-infected individuals. AIDS 22: 489-96.

2. Bruyand M, Thiébaut R, Lawson-Ayayi S, Joly P, Sasco AJ, et al. (2009) Role of uncontrolled HIV RNA level and immunodeficiency in the occurrence of malignancy in HIV-infected patients during the combination antiretroviral therapy era: Agence Nationale de Recherche sur le Sida (ANRS) CO3 Aquitaine Cohort. Clinical Infectious Diseases 49: 1109-16.

3. Mbulaiteye SM, Biggar RJ, Goedert JJ, Engels EA (2003) Immune deficiency and risk for malignancy among persons with AIDS. JAIDS 32: 527-33.

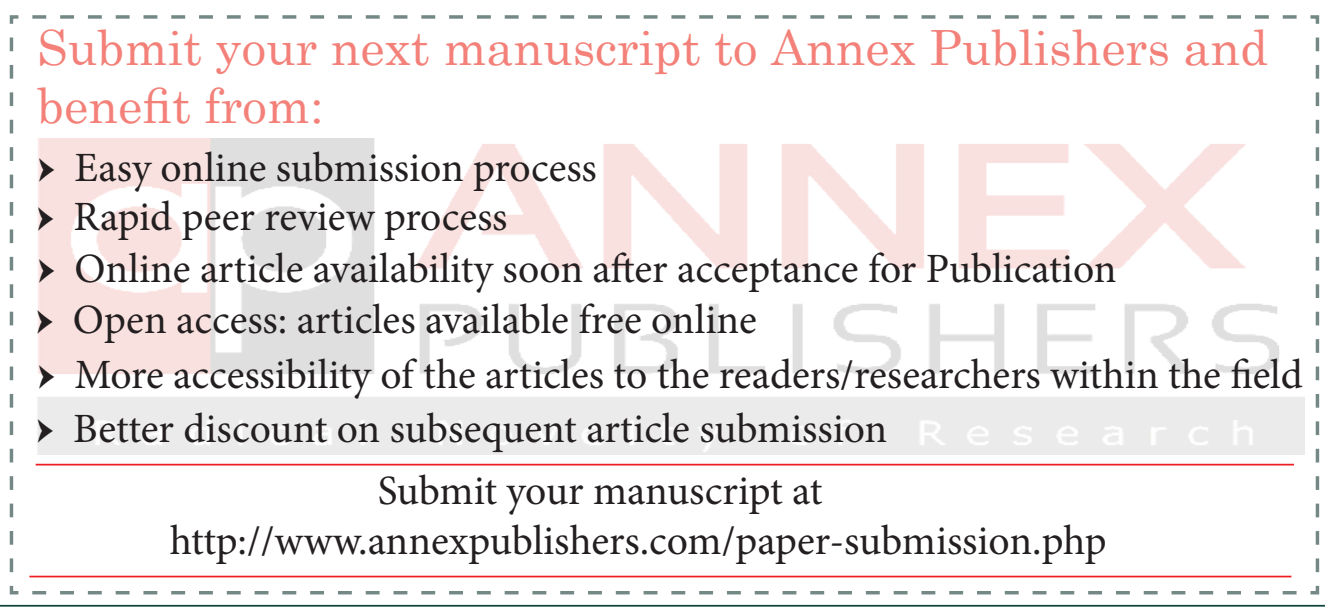

